\title{
General Implicativity in Groupoids
}

\author{
Feng Feng ${ }^{1,2}$ (1) , Hee Sik Kim ${ }^{3, *(1)}$ and Joseph Neggers 4 \\ 1 Department of Applied Mathematics, School of Science, Xi'an University of Posts and Telecommunications, \\ Xi'an 710121, China; fengnix@hotmail.com \\ 2 Shaanxi Key Laboratory of Network Data Analysis and Intelligent Processing, \\ Xi'an University of Posts and Telecommunications, Xi'an 710121, China \\ 3 Department of Mathematics, Research Institute of Natural Sciences, Hanyang University, Seoul 04763, Korea \\ 4 Department of Mathematics, University of Alabama, Tuscaloosa, AL 35487-0350, USA; jneggers@ua.edu \\ * Correspondences: heekim@hanyang.ac.kr; Tel.: +82-10-9276-5630 or +82-2-2220-0897
}

Received: 14 September 2018; Accepted: 29 October 2018; Published: 31 October 2018

check for updates

\begin{abstract}
There are several equivalent axioms, which can be used to characterize the positive implicativity in $B C K$-algebras. In this paper, we investigate interrelationships among such axioms in a more general setting of groupoids, and several aspects regarding their differences in the theory of groupoids.
\end{abstract}

Keywords: groupoid; $d$-algebra; $B C K$-algebra; (positive, central, meet, quasi) implicativity; leftoid; rightoid; (selective, medial) groupoid

\section{Introduction}

Bruck ([1]) published a book, A survey of binary systems discussed in the theory of groupoids, loops and quasigroups, and several algebraic structures. Boruivka ([2]) stated the theory of decompositions of sets and its application to binary systems.

The notion of $d$-algebras which is another useful generalization of $B C K$-algebras was introduced by Neggers and Kim ([3]), and some relations between $d$-algebras and $B C K$-algebras as well as several other relations between $d$-algebras and oriented digraphs were investigated. Several aspects on $d$-algebras were studied [4-6]. Recently some notions of the graph theory were applied to the theory of groupoids ([7]).

There are several different axioms, which can give equivalent characterizations of the positive implicativity in $B C K$-algebras. By using other axioms or their induced results, the proofs of their equivalences were obtained in $B C K$-algebras. It is interesting and useful to investigate these axioms in a more general mathematical structure called groupoids.

In this study, we discuss some relations among such axioms in groupoids, and obtain some results disclosing their differences in the groupoid setting. If we discuss these conditions in $B C K / B C I$-algebras, their delicate differences may not be discovered. Although simple mathematical structures are difficult to deal with in some cases, they can capture essential ideas of some axioms, and provide a starting point of new mathematical structures in future.

\section{Preliminaries}

A groupoid $(X, *)$ is said to be a left-zero-semigroup if $x * y:=x$ for all $x, y \in X$. Similarly, a groupoid $(X, *)$ is said to be a right-zero-semigroup if $x * y:=y$ for all $x, y \in X$. A groupoid $(X, *)$ is said to be a leftoid (resp., rightoid) for $f$ if $x * y:=f(x)$ (resp., $x * y:=f(y)$ ) for a map $f: X \rightarrow X$. For example, if we define a binary operation $*$ by $x * y:=x \sin x$ for all $x, y \in \mathbf{R}$, then $(\mathbf{R}, *)$ is a leftoid [8].

A $d$-algebra [3] is a non-empty set $X$ with a constant 0 and a binary operation " $*$ " satisfying the following axioms: 
(I) $\quad x * x=0$,

(II) $0 * x=0$,

(III) $x * y=0$ and $y * x=0$ imply $x=y$ for all $x, y \in X$.

For brevity, we also call $X$ a $d$-algebra. In $X$, we can define a binary relation " $\leq$ " by $x \leq y$ if and only if $x * y=0$. For general references on $d$-algebras, we refer to [4-6].

A $B C K$-algebra [9] is a $d$-algebra $X$ satisfying the following additional axioms:

(IV) $(x * y) *(x * z)) *(z * y)=0$,

(V) $(x *(x * y)) * y=0$ for all $x, y, z \in X$.

Example $1([6])$. Let $X:=\{0,1,2,3,4\}$ be a set with the following table:

\begin{tabular}{l|lllll}
$*$ & 0 & 1 & 2 & 3 & 4 \\
\hline 0 & 0 & 0 & 0 & 0 & 0 \\
1 & 1 & 0 & 1 & 0 & 1 \\
2 & 2 & 2 & 0 & 3 & 0 \\
3 & 3 & 3 & 2 & 0 & 3 \\
4 & 4 & 4 & 1 & 1 & 0.
\end{tabular}

Then, $(X, *, 0)$ is a d-algebra that is not a BCK-algebra. For general references on BCK-algebras, we refer to [9-11].

A BCK-algebra $(X, *, 0)$ is said to be positive implicative [9] if $(x * z) *(y * z)=(x * y) * z$ for all $x, y, z \in X$.

Theorem 1 ([9]). Let $(X, *, 0)$ be a BCK-algebra. Then, the following are equivalent:

(1) $(X, *, 0)$ is positive implicative,

(2) $x * y=(x * y) * y$,

(3) $(x *(x * y)) *(y * x)=x *(x *(y *(y * x)))$,

(4) $x * y=(x * y) *(x *(x * y))$,

(5) $x *(x * y)=(x *(x * y)) *(x * y)$,

(6) $\quad(x *(x * y)) *(y * x)=(y *(y * x)) *(x * y)$,

for all $x, y, z \in X$.

Let $(X, \leq)$ be a poset and let $S$ be a subset of $X$. A full subposet [12] $(S, \leq)$ is a poset whose underlying set is $S$ and its poset structure is inherited from $(X, \leq)$. A poset $(X, \leq)$ is said to be $Q$-free [13] if there is no full subposet $(P, \leq)$ of $(X, \leq)$ which is order isomorphic to the poset $(Q, \leq)$. Similarly, a graph $(X, \rightarrow)$ is said to be $Q$-free if there is no full subgraph $(P, \rightarrow)$ of $(X, \rightarrow)$ which is isomorphic to the graph $(Q, \rightarrow)$. A directed graph $X:=\left\{x_{1}, x_{2}, \cdots, x_{n}\right\}$ is said to be a cycle of order $n$ if there are arrows $x_{1} \rightarrow x_{2} \rightarrow \cdots \rightarrow x_{n} \rightarrow x_{1}$. We denote it by $C_{n}$. A complete graph is a graph whose vertices are pairwise adjacent, and we denote it by $K_{n}$. For general references on graph theory, we refer to [14].

\section{Positive Implicativity}

A groupoid $(X, *)$ is said to be

- positive implicative: $(x * z) *(y * z)=(x * y) * z$,

- central implicative: $(x * y) * y=x * y$,

- meet implicative: $(x * y) *(x *(x * y))=x * y$,

- quasi implicative: $((x * y) * y) * y=(x * y) * y$,

for all $x, y, z \in X$. 
Proposition 1. (a) If $(X, *)$ is a leftoid for $\varphi$, i.e., $x * y:=\varphi(x)$ for all $x, y \in X$, then $(X, *)$ is positive implicative.

(b) If $(X, *)$ is a rightoid for $\varphi$, i.e., $x * y:=\varphi(y)$ for all $x, y \in X$, then $(X, *)$ is positive implicative if and only if $\varphi^{2}=\varphi$.

Proof. (a). Given $x, y, z \in X$, we have $(x * z) *(y * z)=\varphi(x) * \varphi(y)=\varphi(\varphi(x))=\varphi(x) * z=(x * y) * z$. (b). If $\varphi^{2}=\varphi$, then $(x * z) *(y * z)=\varphi(z) * \varphi(z)=\varphi(\varphi(z))=\varphi(z)=(x * y) * z$, which shows $(X, *)$ is positive implicative. Assume $(X, *)$ is positive implicative. Then, $(x * z) *(y * z)=(x * y) * z$ for all $x, y, z \in X$. It follows that $\varphi(\varphi(z))=\varphi(z) * \varphi(z)=\varphi(z)$, proving that $\varphi^{2}=\varphi$.

Given a graph $(X, \rightarrow)$, a groupoid $(X, *)$ is said to be a selective groupoid from the graph $(X, \rightarrow)$ if

$$
x * y:= \begin{cases}x & \text { if } x \not y \\ y & \text { if } x \rightarrow y\end{cases}
$$

Proposition 2. Let $(X, *)$ be a selective groupoid from a graph $(X, \rightarrow)$. If $(X, *)$ is positive implicative, then $(X, \rightarrow)$ is $C_{3}$-free.

Proof. Assume $(X, \rightarrow)$ has $C_{3}$ as a subgraph. Then, there exist $a, b, c \in X$ such that

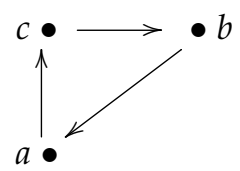

Then, $(a * c) *(b * c)=c * b=b$, while $(a * b) * c=a * c=c$, a contradiction.

Proposition 3. Any selective groupoid $\left(K_{n}, *\right)$ from a complete graph $\left(K_{n}, \rightarrow\right)$ is positive implicative.

Proof. Given $a, b \in K_{n}$, there exist arrows $a \rightarrow b$ and $b \rightarrow a$ in $\left(K_{n}, \rightarrow\right)$, and hence $a * b=b$ and $b * a=a$. It follows that $(a * c) *(b * c)=c * c=c$ and $(a * b) * c=b * c=c$, proving that $\left(K_{n}, *\right)$ is positive implicative.

Proposition 4. Let $(X, *),(Y, \bullet)$ be selective groupoids and let $Z:=X \times Y$. Define $(x, y) \nabla(u, v):=$ $(x * u, y \bullet v)$ for all $(x, y),(u, v) \in Z$. Then,$(Z, \nabla)$ is also positive implicative.

Proposition 5. Let $(X, *)$ be a leftoid for $\varphi$ and let $(Y, \bullet)$ be a rightoid for $\psi$ such that $\psi(y) \bullet \psi(y)=\psi(y)$ for all $y \in Y$. Let $Z:=X \times Y$. Define $(x, y) \nabla(u, v):=(x * u, y \bullet v)$ for all $(x, y),(u, v) \in Z$. Then,$(Z, \nabla)$ is positive implicative.

Proof. Given $(x, y),(u, v) \in Z$, we have $(x, y) \nabla(u, v)=(x * u, y \bullet v)=(\varphi(x), \psi(v))$. It follows that

$$
\begin{aligned}
{\left[\left(x_{1}, y_{1}\right) \nabla(u, v)\right] \nabla\left[\left(x_{2}, y_{2}\right) \nabla(u, v)\right] } & =\left(\varphi\left(x_{1}\right), \psi(v)\right) \nabla\left(\varphi\left(x_{2}\right), \psi(v)\right) \\
& =\left(\varphi\left(x_{1}\right) * \varphi\left(x_{2}\right), \psi(v) \bullet \psi(v)\right) \\
& =\left(\varphi\left(\varphi\left(x_{1}\right)\right), \psi(v)\right),
\end{aligned}
$$

and

$$
\begin{aligned}
{\left[\left(x_{1}, y_{1}\right) \nabla\left(x_{2}, y_{2}\right)\right] \nabla(u, v) } & =\left(\varphi\left(x_{1}\right), \psi\left(y_{2}\right)\right) \nabla(u, v) \\
& =\left(\varphi\left(x_{1}\right) * u, \psi(v) \bullet v\right) \\
& =\left(\varphi\left(\varphi\left(x_{1}\right)\right), \psi(v)\right) .
\end{aligned}
$$


This shows that $(Z, \nabla)$ is positive implicative.

Proposition 6. Let $(X, *)$ be a positive implicative groupoid. If $\varphi:(X, *) \rightarrow(Y, \bullet)$ is an epimorphism of groupoids, then $(Y, \bullet)$ is also positive implicative.

Proof. Given $a, b, c \in Y$, since $\varphi$ is onto, there exist $x, y, z \in X$ such that $a=\varphi(x), b=\varphi, c=\varphi(z)$. It follows that

$$
\begin{aligned}
(a \bullet c) \bullet(b \bullet c) & =(\varphi(x) \bullet \varphi(z)) \bullet(\varphi(y) \bullet \varphi(z)) \\
& =\varphi(x * z) \bullet \varphi(y * z) \\
& =\varphi((x * z) *(y * z)) \\
& =\varphi((x * y) * z) \\
& =\varphi(x * y) \bullet \varphi(z) \\
& =(\varphi(x) \bullet \varphi(y)) \bullet \varphi(z) \\
& =(a \bullet b) \bullet c
\end{aligned}
$$

proving that $(Y, \bullet)$ is also primitive implicative.

Proposition 7. Let $(X, *)$ be a positive implicative groupoid and let $(A, *)$ be a subgroupoid of $(X, *)$. Then, $(A, *)$ is also positive implicative.

By Propositions 5-7, we obtain the following:

Theorem 2. The collection of all positive implicative groupoids forms a variety.

\section{Central and Meet Implicativity}

Proposition 8. Let $(X, *)$ be a leftoid for $\varphi$. Then, $(X, *)$ is central implicative if and only if $\varphi^{2}=\varphi$.

Proof. Assume that $(X, *)$ is central implicative. Then, $(x * y) * y=x * y$ for all $x, y \in X$. It follows that $\varphi(\varphi(x))=\varphi(x) * y=(x * y) * y=x * y=\varphi(x)$, proving that $\varphi^{2}=\varphi$. If $\varphi^{2}=\varphi$, then $(x * y) * y=$ $\varphi(x) * y=\varphi(\varphi(x))=\varphi(x)=x * y$.

Proposition 8 shows that any leftoid $(X, *)$ for $\varphi$, where $\varphi^{2} \neq \varphi$, is positive implicative, but not central implicative.

Example 2. Let $X:=\mathbf{R}$ be the set of all real numbers. Define a binary operation " $*$ " on $X$ by $x * y:=\lfloor x\rfloor$ for all $x, y \in X$. Since $\lfloor\lfloor x\rfloor\rfloor=\lfloor x\rfloor$ for any $x \in X$, the groupoid $(X, *)$ is both positive implicative and central implicative.

Proposition 9. Let $(X, *)$ be a leftoid for $\varphi$. Then, $(X, *)$ is meet implicative if and only if $\varphi^{2}=\varphi$.

Proof. Assume that $(X, *)$ is meet implicative. Then, $(x * y) *(x *(x * y))=x * y$ for all $x, y \in X$. It follows that $\varphi(x) * \varphi(x))=\varphi(x)$ and hence $\varphi^{2}(x)=\varphi(x)$. If $\varphi^{2}=\varphi$, then $(x * y) *(x *(x * y))=$ $\varphi(x) * \varphi(x)=\varphi(\varphi(x))=\varphi(x)=x * y$.

By using Propositions 8 and 9, we obtain the following:

Theorem 3. Let $(X, *)$ be a leftoid for $\varphi$. Then, the following are equivalent:

(i) $(X, *)$ is central implicative,

(ii) $(X, *)$ is meet implicative, 
(iii) $\varphi^{2}=\varphi$.

Example 3. Let $X:=\mathbf{N}$ be the set of all natural numbers. Define a map $\varphi: X \rightarrow X$ by $\varphi(x):=x+1$ and define $x * y:=\varphi(x)$ for all $x, y \in X$. Then, it is easy to see that $(X, *)$ is positive implicative. Since $\varphi(\varphi(x))=$ $\varphi(x+1)=x+2 \neq x+1=\varphi(x)$, by Theorem 3 , it is neither central implicative nor meet implicative.

Proposition 10. Let $(X, *)$ be a rightoid for $\psi$. Then, $(X, *)$ is meet implicative if and only if $\psi^{3}(x)=\psi(x)$ for all $x \in X$.

Proof. Given $x, y \in X$, we have

$$
\begin{aligned}
(X, *) \text { is meet implicative } & \Leftrightarrow(x * y) *(x *(x * y))=x * y \\
& \Leftrightarrow \psi(y) * \psi(x * y)=\psi(y) \\
& \Leftrightarrow \psi(\psi(x * y))=\psi(y) \\
& \Leftrightarrow \psi^{3}(y)=\psi(y),
\end{aligned}
$$

proving the proposition.

Corollary 1. Let $(X, *)$ be a rightoid for $\psi$. If $(X, *)$ is positive implicative, then it is meet implicative.

Proof. Suppose that $(X, *)$ is positive implicative. Then, $(x * y) *(y * z)=(x * y) * z$ for all $x, y, z \in$ $X$. It follows that $\psi(z) * \psi(z)=\psi(z)$ and hence $\psi(\psi(z))=\psi(z)$ for all $z \in X$. This shows that $\psi(\psi(\psi(z)))=\psi(\psi(z))=\psi(z)$ for all $z \in X$. By Proposition $10,(X, *)$ is meet implicative.

The converse of Corollary 1 does not hold in general.

Example 4. Consider $X:=\mathbf{Z} /(6)$. If we define $\psi(x+(6)):=x+3+(6)$ for all $x+(6) \in X$, and define $(x+(6)) *(y+(6)):=\psi(y+(6))$ on $X$, then $(X, *)$ is a rightoid for $\psi$. Given $x+(6) \in X$, we have $\psi(\psi(x+(6)))=\psi(x+3+(6))=x+3+3+(6)=x+(6) \neq x+3+(6)=\psi(x+(6))$ and $\psi(\psi(\psi(x+(6))))=\psi(\psi(x+3+(6)))=\psi(x+3+3+(6))=x+3+3+3+(6)=x+3+(6)=$ $\psi(x+(6))$. By Theorem 3 and Corollary $1,(X, *)$ is meet implicative, but not positive implicative.

Note that a central implicative groupoid need not be a meet implicative groupoid. Note that every rightoid $(X, *)$ for $\psi$ is central implicative, since $(x * y) * y=\psi(y)=x * y$ for all $x, y \in X$. By Corollary 1 , we obtain that every positive implicative rightoid $(X, *)$ for $\psi$ is both central implicative and meet implicative.

Example 5. Define a binary operation " $*$ " on $X:=\mathbf{Z} /(6)$ by $(x+(6)) *(y+(6)):=\psi(y+(6))$ where $\psi(x+(6)):=x+1+(6)$. Then, $(X, *)$ is a rightoid for $\psi$. Given $x+(6) \in X$, we have $\psi(\psi(\psi(x+$ $(6))))=x+3+(6) \neq x+1+(6)=\psi(x+(6))$. This shows that $(X, *)$ is central implicative, but not meet implicative.

Proposition 11. Every selective groupoid is both central implicative and meet implicative.

Proof. Let $(X, *)$ be a selective groupoid. Given $x, y \in X$, if $x * y=x$, then $(x * y) * y=x *$ $y=x=x * y$. If $x * y=y$, then $(x * y) * y=y * y=y=x * y$, proving that $(X, *)$ is central implicative. Given $x, y \in X$, if $x * y=x$, then $(x * y) *(x *(x * y))=x *(x * x)=x=x * y$. If $x * y=y$, then $(x * y) *(x *(x * y))=(x * y) *(x * y)=y * y=y=x * y$, proving that $(X, *)$ is meet implicative.

Note that selective groupoids need not be positive implicative. 
Example 6. Let $X:=\{x, y, z\}$ be a set with the following graph:

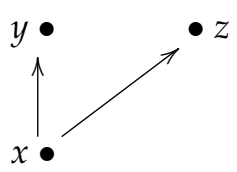

Then, its selective groupoid $(X, *)$ can be represented as follows:

\begin{tabular}{l|lll}
$*$ & $x$ & $y$ & $z$ \\
\hline$x$ & $x$ & $y$ & $z$ \\
$y$ & $y$ & $y$ & $y$ \\
$z$ & $z$ & $z$ & $z$
\end{tabular}

Then, it is easy to see that $(X, *)$ is both central implicative and meet implicative. Since $(x * z) *(y * z)=$ $z * y=z \neq y=y * z=(x * y) * z$, it is not positive implicative.

Example 7. Let $(X, *)$ be a leftoid for $\varphi$ and $|X| \geq 2$. If $\varphi(x)=t_{0} \in X$ for all $x \in X$, then $\varphi(\varphi(x))=$ $\varphi\left(t_{0}\right)=t_{0}=\varphi(x)$, i.e., $(X, *)$ is both central implicative and meet implicative. Note that $\left(X, *, t_{0}\right)$ need not be a d/BCK-algebra. In fact, assume $x * y=y * x=t_{0}$. Then, $\varphi(x)=\varphi(y)=t_{0}$. Since $|X| \geq 2, x=y$ does not hold in general.

\section{Quasi Implicativity}

Proposition 12. Let $(X, *)$ be a leftoid for $\varphi$. Then, $(X, *)$ is quasi implicative if and only if $\varphi^{3}=\varphi^{2}$.

Proof. Assume that $(X, *)$ is quasi implicative. Then, $((x * y) * y) * y=(x * y) * y$ for all $x, y \in X$. It follows that $\varphi(\varphi(\varphi(x)))=\varphi(\varphi(x * y))=\varphi((x * y) * y)=((x * y) * y) * y=(x * y) * y=\varphi(x * y)=$ $\varphi(\varphi(x))$, proving that $\varphi^{3}=\varphi^{2}$.

If $\varphi^{3}=\varphi^{2}$, then $((x * y) * y) * y=\varphi^{3}(x)=\varphi^{2}(x)=(x * y) * y$, proving that $(X, *)$ is quasi implicative.

It is clear that every central implicative groupoid is quasi implicative, but the converse need not be true in general.

Example 8. Let $X:=\{0,1,2\}$ be a set with $x * y:=\varphi(x)$ for all $x, y \in X$ where $\varphi(0)=\varphi(1)=0, \varphi(2)=1$. Then, $\varphi^{2}(0)=\varphi^{2}(1)=\varphi^{2}(2)=0, \varphi(2)=1 \neq 0=\varphi^{2}(2)$ and $\varphi^{3}(x)=0=\varphi^{2}(x)$ for all $x \in X$. By Theorem 3 and Proposition 12, $(X, *)$ is quasi implicative, but not central (meet) implicative.

Proposition 13. Every rightoid for $\psi$ is both central implicative and quasi implicative.

Proof. Given $x, y \in X$, we have $(x * y) * y=((x * y) * y) * y=x * y=\psi(y)$, proving that $(X, *)$ is both central implicative and quasi implicative.

\section{Medial and Positive Implicative Groupoids}

A groupoid $(X, *)$ is said to be medial if $(x * y) *(u * v)=(x * u) *(y * v)$ for all $x, y, u, v \in X$.

Example 9. Let $X:=\mathbf{R}$ be the set of all real numbers. If we define $x * y:=\max \{x, y\}$ for all $x, y \in X$, then $(X, *)$ is both medial and idempotent. It is also a positive implicative groupoid. 
Example 10. Let $X:=\mathbf{R}$ be the set of all real numbers and let $\alpha \in X$. Define a map $\varphi: X \rightarrow X$ and define a binary operation " $*$ " on $X$ by $x * y:=\alpha+\varphi(x)$ for all $x, y \in X$. Then, $(X, *)$ is medial. In fact, given $x, y, u, v \in X$, we have

$$
\begin{aligned}
(x * y) *(u * v) & =\alpha+\varphi(x * y) \\
& =\alpha+\varphi(\alpha+\varphi(x)) \\
& =\alpha+\varphi(x * u) \\
& =(x * u) *(y * v),
\end{aligned}
$$

showing that $(X, *)$ is medial.

Example 11. In Example 10, if $(X, *)$ is idempotent, then $x * x=x$ for all $x \in X$, which shows that $\alpha+\varphi(x)=x$, i.e., $\varphi(x)=x-\alpha$ for all $x \in X$.

Note that if $(X, *)$ is idempotent in Examples 10 and 11, then $x * y=\alpha+\varphi(x)=\alpha+(x-\alpha)=x$ for all $x, y \in X$. This shows that $(X, *)$ is a left-zero semigroup.

Proposition 14. If $(X, *)$ is an idempotent medial groupoid, then it is positive implicative.

Proof. If $(X, *)$ is medial and positive implicative, then for any $x, y, z \in X$, we have $(x * z) *(y * z)=$ $(x * y) *(z * z)=(x * y) * z$, proving that $(X, *)$ is positive implicative.

Note that the converse of Proposition 14 need not be true in general.

Example 12. Let $X:=\{0, x, y, u, v\}$ be a poset with the following Hasse diagram:

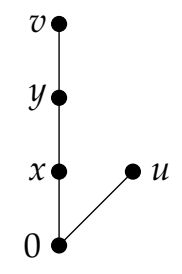

If we define a binary operation "*" on X by

$$
x * y:= \begin{cases}0 & \text { if } x \leq y, \\ x & \text { if } x \leq \leq y\end{cases}
$$

then it is easy to see that $(X, *, 0)$ is a positive implicative $B C K$-algebra. Note that $(x * y) *(u * v)=0 * u=0$, but $(x * u) *(y * v)=x * 0=x$, which shows that $(X, *)$ is not medial. Since $x * x=0 \neq x$ for all $x \in X \backslash\{0\}$, it is not idempotent.

\section{Conclusions}

In this paper, we proved that the collection of all positive implicative groupoids forms a variety, and discussed some relations between selective groupoids and the graph theory. We ascertained several relations between the central implicativity and the meet implicativity in groupoids. Moreover, we showed that every selective groupoid is both central and meet implicative, and every idempotent groupoid is positive implicative. For further investigation, we will apply this concept to the semigroup $\operatorname{Bin}(X)([8])$ of all groupoids defined on a set $X$ related to ordered structures in groupoids ([15]).

Author Contributions: Conceptualization, F.F., H.S.K. and J.N.; Formal analysis, F.F., H.S.K. and J.N.; Writing—original draft, F.F., H.S.K.; Writing—review \& editing, F.F., H.S.K. and J.N. 
Funding: This work was partially supported by National Natural Science Foundation of China (Program No. 11301415), Natural Science Basic Research Plan in Shaanxi Province of China (Program No. 2018JM1054), Scientific Research Program Funded by Shaanxi Provincial Education Department of China (Program No. 16JK1696), and the Special Funds Project for Key Disciplines Construction of Shaanxi Universities.

Acknowledgments: The authors are deeply grateful to four anonymous reviewers for their valuable suggestions.

Conflicts of Interest: The author declares no conflicts of interest.

\section{References}

1. Bruck, R.H. A Survey of Binary Systems; Springer: Berlin, Germany, 1958.

2. Borůvka, O. Foundations of the Theory of Groupoids and Groups; John Wiley \& Sons: New York, NY, USA, 1976.

3. Neggers, J.; Kim, H.S. On d-algebras. Math. Slovaca 1999, 49, 19-26.

4. Allen, P.J.; Kim, H.S.; Neggers, J. On companion d-algebras. Math. Slovaca 2007, 57, 93-106. [CrossRef]

5. Allen, P.J.; Kim, H.S.; Neggers, J. Deformations of $d /$ BCK-algebras. Bull. Korean Math. Soc. 2011, 48, 315-324. [CrossRef]

6. Neggers, J.; Jun, Y.B.; Kim, H.S. On $d$-ideals in $d$-algebras. Math. Slovaca 1999, 49, $243-251$.

7. Kim, H.S.; Neggers, J.; Ahn, S.S. A method to identify simple graphs by special binary systems. Symmetry 2018, 10, 297. [CrossRef]

8. Kim, H.S.; Neggers, J. The semigroups of binary systems and some perspectives. Bull. Korean Math. Soc. 2008, 45, 651-661. [CrossRef]

9. Meng, J.; Jun, Y.B. BCK-Algebras; Kyungmoon Sa: Seoul, Korea, 1994.

10. Huang, Y. BCI-Algebras; Science Press: Beijing, China, 2006.

11. Iorgulescu, A. Algebras of Logic as BCK-Algebras; Editura ASE: Bucharest, Romania, 2008.

12. Neggers, J.; Kim, H.S. Basic Posets; World Scientific Pub. Co.: Singapore, 1998.

13. Neggers, J.; Kim, H.S. Modular posets and semigroups. Semigroup Forum 1996, 53, 57-62. [CrossRef]

14. West, D.B. Introduction to Graph Theory; Prentice Hall: Upper Saddle River, NJ, USA, 2001.

15. Kim, H.S.; Neggers, J.; So, K.S. Order related concepts for arbitrary groupoids. Bull. Korean Math. Soc. 2017, $54,1373-1386$.

(C) 2018 by the authors. Licensee MDPI, Basel, Switzerland. This article is an open access article distributed under the terms and conditions of the Creative Commons Attribution (CC BY) license (http:/ / creativecommons.org/licenses/by/4.0/). 